\title{
DIVERSITY, EUROPEAN IDENTITY AND SOCIAL PSYCHOLOGICAL KNOWLEDGE
}

\author{
Yolanda Zografova \\ IPHS, Bulgarian Academy of Sciences, Bulgaria \\ E-mail: zografova@abv.bg
}

The wide range of transformations subsequent to the enlargements of the European community reverberate in all important spheres of the way of life. The individual and social psychic experience the important influence of the enlargement processes and so do the interhuman, intergroup and cross-cultural relations. To a mutual intergroup tolerance and the lacking conflicts integration of foreign citizens, of immigrants and refugees in a certain country, lead the importance of a collective European identity and the formation of commonly shared values, norms and rules. This is found to be a controversial and uneasy process. Social knowledge and social psychology in particular could help elaborate new models of relations on a supra-individual level directed toward research on collective phenomena through interdisciplinary approach.

Modern processes of globalization and integration have great impact and change social psychic on national, community and group level. Nevertheless the dynamics in development of interpersonal and intergroup relations, there is a problem that remains crucial - the identity problem. Furthermore, the higher the integration of the communities, the sharper the outlined identification parameters. Phenomena as increased mobility, developed communication opportunities, migration flows, refugees, determine formation of new attitudes, new representations and new forms of social behavior among European citizens. However, some of the social development trends are unpredictable and this leads to unscheduled changes in social relations and intergroup tolerance. Multiculturalism that should have been a theory and at the same time real social context so as communities with their specificities and diversity to coexist in mutual tolerance, could not overcome the intergroup conflicts (Putnam, 2007) and was even accused of stimulating intolerance. Thus, in the field of social psychology one of the most often quoted and seemingly most often confirmed theory remains Tajfel's TSI (Tajfel, 1981) with the clear positioning of We and They, of others and ours. However, Druckman suggests that studies should develop toward analysis of the reality of multiple and mutually crossing identities that are intrinsic to to contemporary individuals (Druckman, 2001).

Social processes provoked new challenges both for politicians and social knowledge, including psychology. Diversity paradigm could be one unifying idea for the different branches and it is approached by some of the social sciences, however it still rarely is in the focus of psychological knowledge. This paradigm allows one's own Self to be viewed as one of the Others and approach to harmonization of the multiple systems to which person relates to be outlined (Sicakkan, 2003).

Acceptance of the ethnical, national, communal diversity on individual and collective level could be the factor for development of intergroup tolerance. When speaking of European citizens in particular, at the foreground comes also the issue of the common, collective European identity. Nevertheless the number of authors who are sceptical to its formation, awareness of the common affiliation to the EU is obviously outlined as the basis, necessary for overcoming many social and economic issues, as well as for attainment of the important adaptation, integration of the foreigners, immigrants, etc. in one state or another. Increasing the European citizens' awareness about the principles, promulgated through EC instruments such as the Lisbon treaty or about the opportunities of European Citizens Initiative facilitates development of common normative and value models, but 
this is not sufficient. Being well informed about the general principles, rules, and norms is not always related to specific forms of behavior. This is confirmed also by the data of the representative large Volume 7, 2013 scale ESS on the attitudes towards foreign groups of immigrants in European countries. It turns out that dominating are the positive attitudes to immigration and its general effect on economy, culture and life in the countries; however at the same time there are some ambiguous attitudes, i.e. among European citizens, the willingness immigrants to be accepted is not well expressed. This means that the positive perception of the influence immigration has on the various aspects of the social economic and cultural life is not always related to attitudes the respective groups of immigrants to be accepted (Zografova, 2010).

The contemporary situation of high mobility and immigration is common in a lot of European countries and often conflicts erupt between representatives of different ethnic groups especially when socially unequal people devoted to pursue their personal status and employment are involved, which contradicts to the interests of the local nationals. This provokes some reactions and actions immigrants to be excluded in the different countries, which in essence is the contradiction to the accepted common values and outlines ambivalence in the attitudes - acceptance of the effects of immigration as positive, but rejection of the representatives of foreign and ethnic groups, especially when it comes to competition. Acceptance of the ethnonational diversity and development of broader representations, including the others' identity in the general collective one, incorporating „Them “ to „Us" is a complicated process. Social cognition and social psychology in particular could facilitate analysis and solving the new problems, originating in Community integration and expansion. A need has been sought to develop new concepts and research approaches to the problems at societary and collective, supra-individual and also supra-national level in social- psychological perspective. This leads to the necessity of interdisciplinarity in the analysis of the complicate and dynamically changing collective phenomena.

\section{References}

Druckman, D. (2001). Nationalism and War: A Social Psychological Perspective. In D. Christie, R. Wagner \& D. Winter (Eds.), Peace, Conflict, and Violence: Peace Psychology for the $21^{\text {st }}$ Century. (pp.1-30), Englewood Cliffs, New Jersey: Prentice-Hall, Psychology Book, Retrieved from http://academic.marion. ohio-state.edu/dchristie/Peace

Putnam, R., (2007). E Pluribus Unum: Diversity and Community in the Twenty-first Century. The 2006 Johan Skytte Prize Lecture. Scandinavian Political Studies, 30 (2), 137-174.

Sicakkan, H. (2003). Politics, Wisdom and Diversity. Or Why I don't want to be tolerated. Norwegian Journal of Migration Research, 1, 32-44.

Tajfel, H. (1981). Human groups and social categories: studies in Social Psychology. Cambridge

Zografova, Y. (2010). Social Psychological Dimensions of Otherness. Challenges before social psychological knowledge. Bulgarian Journal of Psychology, 1-4, 263-273.

Received: October 01, 2013

Accepted: November 15, 2013 